High-mass X-ray binaries: illuminating the passage from massive

binaries to merging compact objects

Proceedings IAU Symposium No. 346, $2018 \quad$ C) 2018 International Astronomical Union

A.C. Editor, B.D. Editor \& C.E. Editor, eds.

DOI: 00.0000/X000000000000000X

\title{
Studying the presence of magnetic fields in a sample of high-mass X-ray binaries
}

\author{
Swetlana Hubrig ${ }^{1}$, Alexander F. Kholtygin ${ }^{2}$, Lara Sidoli ${ }^{3}$, \\ Markus Schöller ${ }^{4}$, and Silva P. Järvinen ${ }^{1}$ \\ ${ }^{1}$ Leibniz-Institut für Astrophysik Potsdam (AIP), An der Sternwarte 16, 14482 Potsdam, \\ Germany, email: shubrig@aip.de \\ ${ }^{2}$ Saint-Petersburg State University, Universitetskij pr. 28, 198504 Saint-Petersburg, Russia \\ ${ }^{3}$ INAF, Istituto di Astrofisica Spaziale e Fisica Cosmica, Via E. Bassini 15, 20133 Milano, Italy \\ ${ }^{4}$ European Southern Observatory, Karl-Schwarzschild-Str. 2, 85748 Garching, Germany
}

\begin{abstract}
Previous circular polarization observations obtained with the ESO FOcal Reducer low dispersion spectrograpgh at the VLT in 2007-2008 revealed the presence of a weak longitudinal magnetic field on the surface of the optical component of the X-ray binary Cyg X-1, which contains a black hole and an O9.7Iab supergiant on a $5.6 \mathrm{~d}$ orbit. In this contribution we report on recently acquired FORS 2 spectropolarimetric observations of Cyg X-1 along with measurements of a few additional high-mass X-ray binaries.
\end{abstract}

Keywords. stars: magnetic fields, stars: individual (BP Cru, Cyg X-1, Vela X-1, LS 5039), (stars:) supergiants, (stars:) binaries: general, X-rays: stars

\section{Introduction}

High-mass X-ray binaries are fundamental for studying stellar evolution, nucleosynthesis, structure and evolution of galaxies, and accretion processes. The classical high-mass $\mathrm{X}$-ray binaries (HMXBs) with supergiant companions (SgHMXBs) are known since the birth of X-ray astronomy and are persistent X-ray emitters, with a limited range of intensity variability (around a factor of 10). These targets are among the brightest X-ray sources in the sky. For them, the observed spectral and time variability is best explained by assuming that accretion onto the compact object is taking place from a highly structured stellar wind, where cool dense clumps are embedded in a rarefied photoionized gas.

While the first spectropolarimetric observations using the FOcal Reducer low dispersion Spectrograph (FORS 1/2; Appenzeller et al. 1998) mounted on the $8 \mathrm{~m}$ Antu telescope of the Very Large Telescope indicated the presence of a rather strong longitudinal magnetic field of a kG order in the supergiant fast X-ray transient (SFXT) IGR J112155952 (Hubrig et al. 2018), no systematical search for magnetic fields was carried out in SgHMXBs. SFXTs are a subclass of HMXBs associated with early-type supergiant companions, and characterized by sporadic, short and bright Xray flares reaching peak luminosities of $10^{36}-10^{37} \mathrm{erg} \mathrm{s}^{-1}$ and typical energies released in bright flares of about $10^{38}-10^{40} \mathrm{erg}$ (see the review by Sidoli in 2017 for more details). Different accretion regimes - transient in the settling accretion mode versus persistent in the free-fall Bondi mode - were suggested in the last years for SFXTs and SgHMXBs, respectively.

The magnetic field of the eclipsing binary VelaX-1 with a B0.5Ia component and an orbital period of $8.96 \mathrm{~d}$ was previosly studied by Hubrig et al. (2013). However, no magnetic field detection at a significance level of $3 \sigma$ has been achieved in this system. The spectral behaviour of Vela X-1 is known to be very complex due to the presence of 


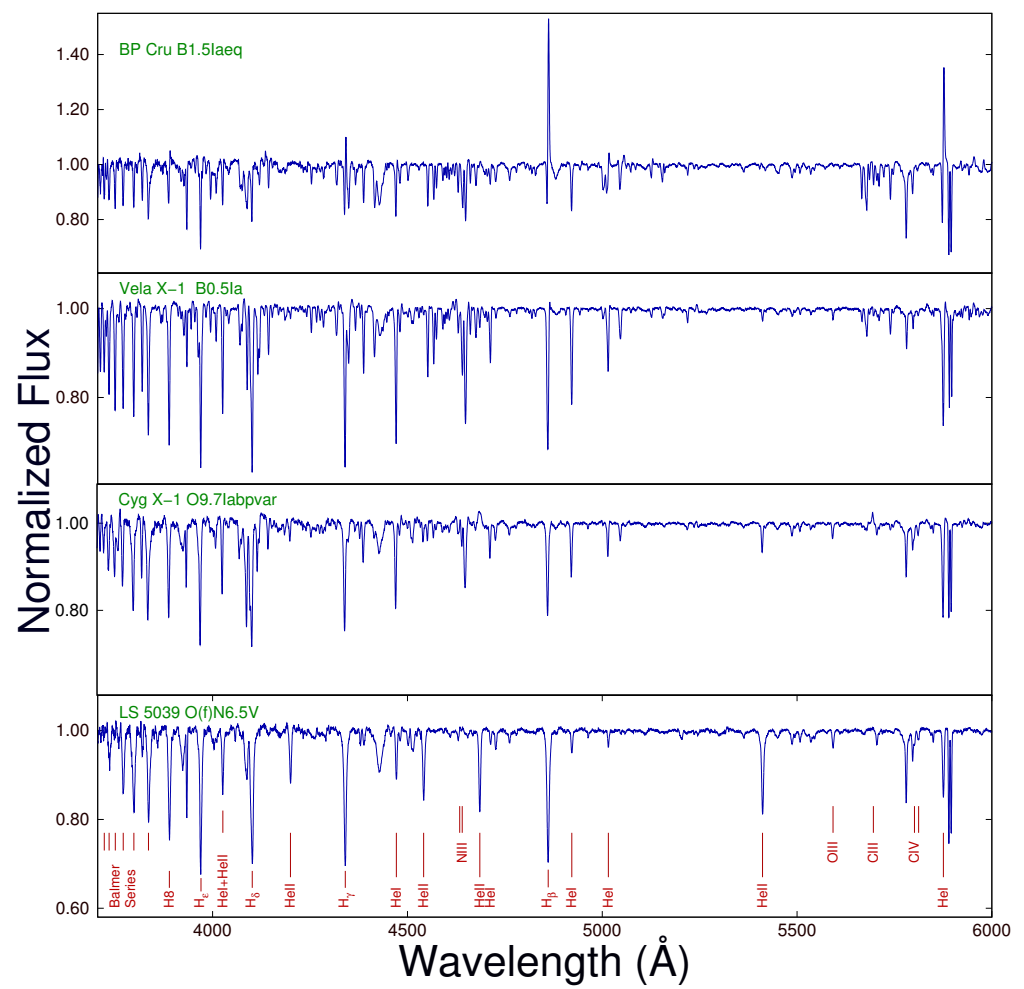

Figure 1. Normalised FORS 2 spectra of LS 5039, Cyg X-1, Vela X-1, and BP Cru. Well known spectral lines are indicated.

bumps and wiggles in the line profiles, and an impact of tidal effects producing orbital phase-dependent variations in the line profiles leading to asymmetries such as extended blue or red wings (Koenigsberger et al. 2012). Furthermore, Kreykenbohm et al. (2008) detected flaring activity and temporary quasi-periodic oscillations in INTEGRAL Xray observations. Magnetic field measurements of the X-ray binary Cyg X-1, with the historically first black-hole candidate, using FORS 2 low-resolution spectropolarimetric observations were reported by Karitskaya et al. (2010). The authors detected a relatively weak mean longitudinal magnetic fields of the order of $100 \mathrm{G}$ with a few measurements at a significance level in the range between 3.5 and $6.2 \sigma$.

In this contribution we discuss the most recent FORS 2 spectropolarimetric observations of the Cyg X-1 system and two other SgHMXB systems, BP Cru, and LS 5039. For completeness we also present the older results for Vela X-1, as this system can be considered as the prototype of persistent HMXBs.

\section{Magnetic field measurements}

The FORS 2 multi-mode instrument is equipped with polarisation analysing optics comprising super-achromatic half-wave and quarter-wave phase retarder plates, and a Wollaston prism with a beam divergence of $22^{\prime \prime}$ in standard resolution mode. We used the GRISM $600 \mathrm{~B}$ and the narrowest available slit width of $0.4^{\prime \prime}$ to obtain a spectral resolving power of $R \approx 2000$. The observed spectral range from 3250 to $6215 \AA$ includes all Balmer lines, apart from $\mathrm{H} \alpha$, and numerous helium lines. For the observations, we used a non-standard readout mode with low gain $(200 \mathrm{kHz}, 1 \times 1$,low $)$, which provides a broader 
Table 1. Longitudinal magnetic field values obtained using FORS 2 observations of four highmass X-ray binaries. In the first two columns we show the name of the binary and the modified Julian date of mid-exposure, followed by the mean longitudinal magnetic field using the Monte Carlo bootstrapping test, for all lines and for the hydrogen lines. In the last column, we present the significance of the measurements of $\left\langle B_{\mathrm{z}}\right\rangle_{\text {all }}$ using the set of all lines. All quoted errors are $1 \sigma$ uncertainties.

\begin{tabular}{ccccc}
\hline \hline Name & MJD & $\begin{array}{c}\left\langle B_{\mathrm{z}}\right\rangle_{\text {all }} \\
{[\mathrm{G}]}\end{array}$ & $\begin{array}{c}\left\langle B_{\mathrm{z}}\right\rangle_{\text {hyd }} \\
{[\mathrm{G}]}\end{array}$ & $\begin{array}{c}\text { Significance } \\
\end{array}$ \\
& & \multicolumn{1}{c}{$\sigma$} & \multicolumn{1}{c}{$\sigma$} \\
\hline BP Cru & 57528.0781 & $344 \pm 149$ & $441 \pm 173$ & 2.3 \\
BP Cru & 57533.1965 & $-144 \pm 162$ & $-319 \pm 193$ & 0.9 \\
BP Cru & 57591.0529 & $254 \pm 162$ & $82 \pm 214$ & 1.6 \\
Cyg X-1 & 57585.1653 & $159 \pm 63$ & $447 \pm 221$ & 2.5 \\
Cyg X-1 & 57593.2230 & $-61 \pm 82$ & $-274 \pm 237$ & 0.8 \\
Cyg X-1 & 57644.0254 & $-88 \pm 44$ & $-111 \pm 157$ & 2.0 \\
Cyg X-1 & 57645.0189 & $-79 \pm 45$ & $-52 \pm 114$ & 1.7 \\
Vela X-1 & 55686.0962 & $-15 \pm 30$ & $-11 \pm 45$ & 0.5 \\
Vela X-1 & 55687.0584 & $-80 \pm 32$ & $-114 \pm 50$ & 2.5 \\
Vela X-1 & 55688.0479 & $57 \pm 37$ & $88 \pm 69$ & 1.5 \\
LS 5039 & 57585.1260 & $794 \pm 277$ & $634 \pm 317$ & 2.5 \\
LS 5039 & 57591.0970 & $-517 \pm 291$ & $-118 \pm 383$ & 1.8 \\
LS 5039 & 57646.1463 & $534 \pm 268$ & $199 \pm 340$ & 2.0 \\
LS 5039 & 57611.2456 & $525 \pm 450$ & $953 \pm 617$ & 1.2 \\
\hline
\end{tabular}
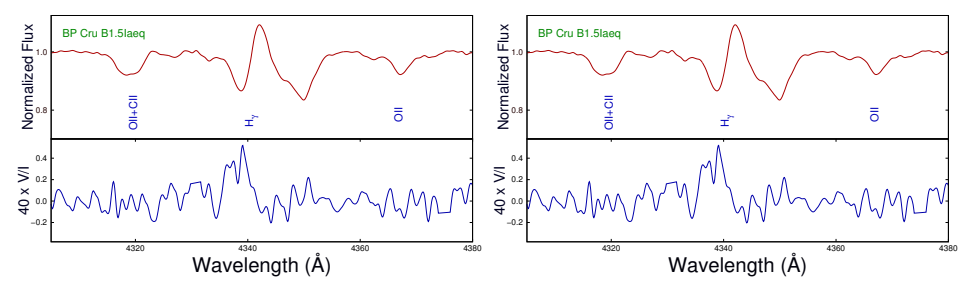

Figure 2. Examples of Stokes $I$ and Stokes $V$ spectra of BP Cru (left panel) and Cyg X-1 (right panel) in the vicinity of the $H \gamma$ line and the He I 5876 line, respectively.

dynamic range, hence allowed us to reach a higher signal-to-noise ratio in the individual spectra. The spectral appearance of all targets in the FORS 2 spectra is presented in Fig. 11

The longitudinal magnetic field was measured in two ways: using the entire spectrum including all available lines or using exclusively the hydrogen lines. Furthermore, we carried out Monte Carlo bootstrapping tests. These are most often applied with the purpose of deriving robust estimates of standard errors. The measurement uncertainties obtained before and after the Monte Carlo bootstrapping tests were found to be in close agreement, indicating the absence of reduction flaws. The results of our magnetic field measurements, those for the entire spectrum or only for the hydrogen lines, are presented in Table 1

Our measurements do not reveal the presence of significant mean longitudinal magnetic fields in any of the four studied binaries. We observe changes of the field polarity in the measurements of all targets, but the measurement uncertainties are rather large, leading to significance levels of only $2.3-2.5 \sigma$ and less. On the other hand, as we show in Fig. 2 . typical Zeeman features are detected in the FORS 2 Stokes $V$ spectra of BP Cru and Cyg X-1. 

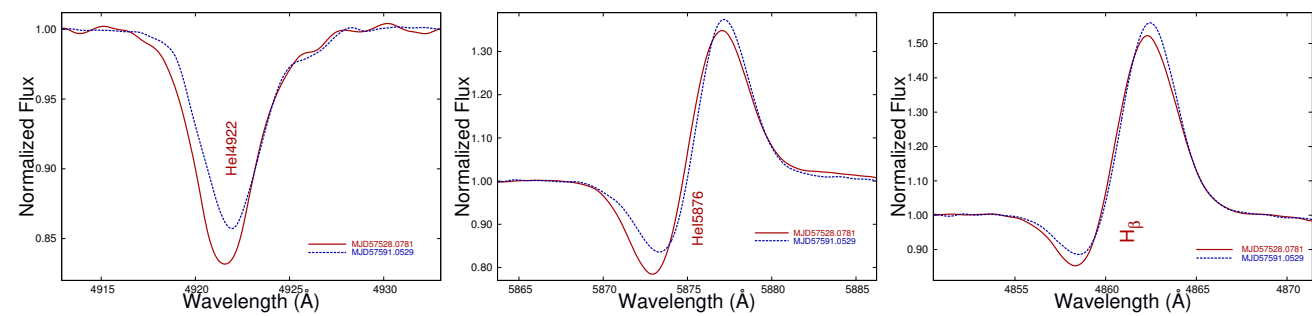

Figure 3. Variability of spectral lines in the FORS 2 spectra of BP Cru recorded on two different nights.
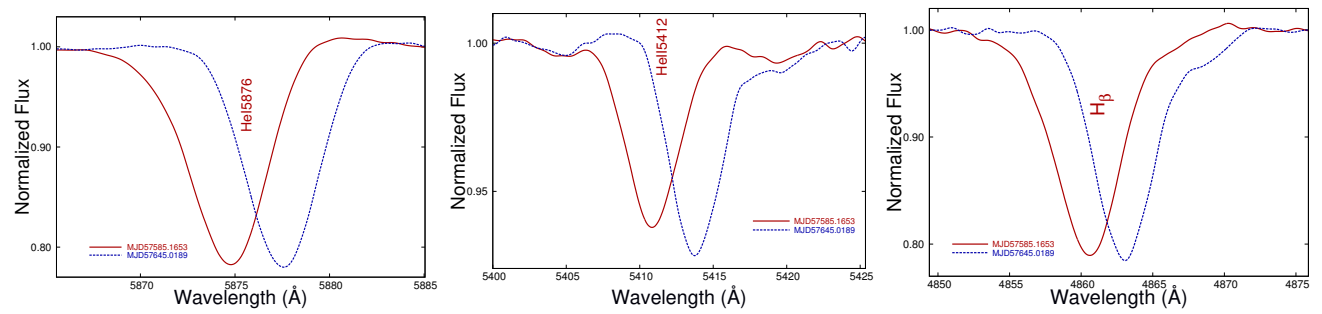

Figure 4. Same as in Fig. 3, but for Cyg X-1.
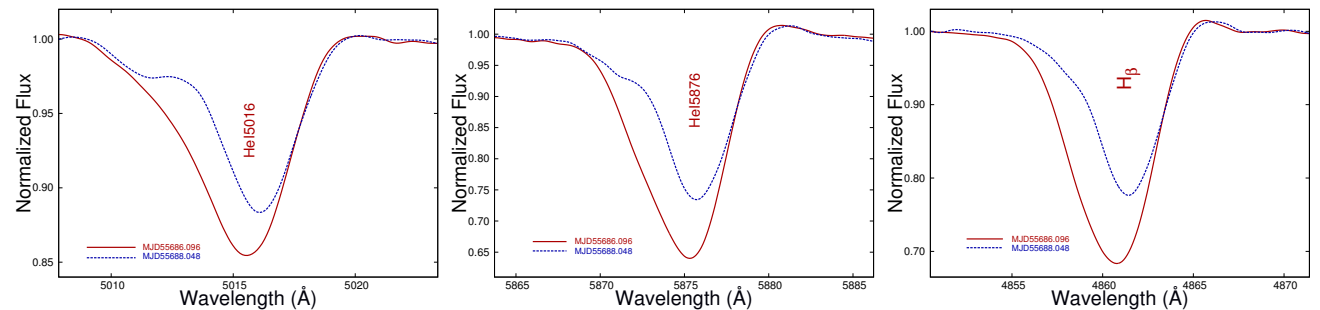

Figure 5. Same as in Fig. 3 but for Vela X-1.

\section{Discussion}

The recent spectropolarimetric observations of four SgHMXBs with FORS 2 showed changes in the field polarities, but the measurement uncertainties are too large to allow us to conclude on the presence of magnetic fields. Apart from the search for magnetic fields, the acquired spectra of the optical components allowed us to detect significant spectral variability: spectral lines belonging to hydrogen and other elements show changes of the line intensities and radial velocities over different observing nights. We present a few examples in Figs. 3 [6 showing individual Stokes $I$ helium and hydrogen line profiles. It is not clear yet whether the detected spectral variability is caused by the presence of magnetospheres or by pulsational variability, frequently detected in massive OB-type stars. Future observations are urgently needed to be able to draw solid conclusions about the role of magnetic fields in these targets.

\section{References}

Appenzeller, I., Fricke, K., Fürtig, W., Gässler, W., Häfner, R., Harke, R., Hess, H.-J., Hummel, W., Jürgens, P., Kudritzki, R.-P., Mantel, K.-H., Meisl, W., Muschielok, B., Nicklas, H., Rupprecht, G., Seifert, W., Stahl, O., Szeifert, T., \& Tarantik, K. 1998, The ESO Messenger, 94, 1

Hubrig, S., Schöller, M., Ilyin, I., Kharchenko, N.V., Oskinova, L.M., Langer, N., González, J.F., Kholtygin, A.F., Briquet, M., \& Magori Collaboration 2013, A\&A, 551, A33 

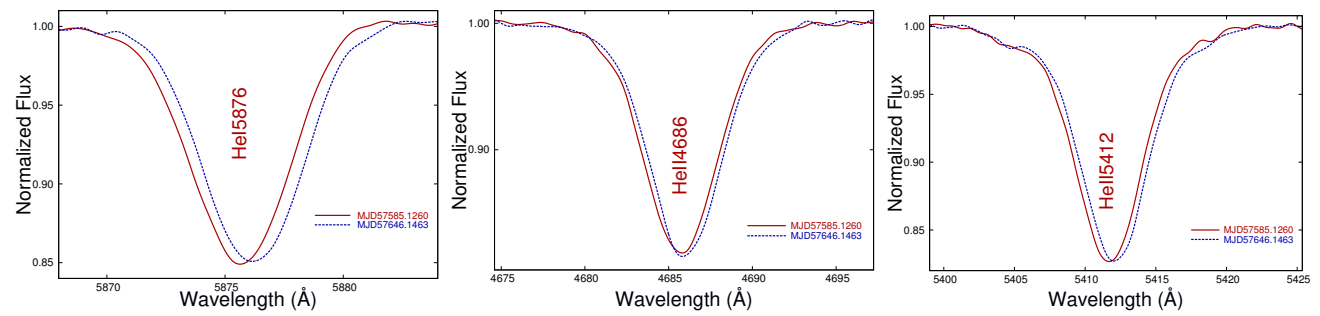

Figure 6. Same as in Fig. 3 but for LS 5039.

Hubrig, S., Sidoli, L., Postnov, K., Schöller, M., Kholtygin, A.F., Järvinen, S.P., \& Steinbrunner, P. 2018, MNRAS, 474, L27

Karitskaya, E.A., Bochkarev, N.G., Hubrig, S., Gnedin, Y.N., Pogodin, M.A., Yudin, R.V., Agafonov, M.I., \& Sharova, O.I. 2010, IBVS, 5950, 1

Koenigsberger, G., Moreno, E., \& Harrington, D.M. 2012, A 6 A, 539, A84

Kreykenbohm, I., Wilms, J., Kretschmar, P., Torrejón, J.M., Pottschmidt, K., Hanke, M., Santangelo, A., Ferrigno, C., \& Staubert, R. 2008, A\& A, 492, 511

Sidoli, L. 2017, Proc. of the "XII Multifrequency Behaviour of High Energy Cosmic Sources Workshop", 12-17 June, 2017 Palermo, Italy, 52 